\title{
Casos prácticos propuestos por el alumnado en Psicometría
}

\section{Practical cases proposed by the students in Psychometrics}

SUSANA SANDUVETE CHAVES

ORCID: https://orcid.org/0000-0001-8568-6168

Universidad de Sevilla

Facultad de Psicología

Departamento de Psicología Experimental

sussancha@us.es

Fecha de recepción: 19/11/2019

Fecha de aceptación: 25/11/2019

DOI: http://dx.doi.org/10.12795/9788447221912.117

Pp.: 2599-2612 


\section{Resumen}

Habitualmente, el alumnado de la asignatura Psicometría (grupo en inglés), de tercero del Grado en Psicología, dedicaba las clases prácticas a resolver ejercicios propuestos por el profesorado y luego participar en la corrección de éstos, en una situación donde era la profesora quien llevaba la voz cantante. El objetivo de este Ciclo de Mejora en el Aula fue el de fomentar la participación del alumnado, tanto en la elaboración de ejercicios como en la resolución de éstos, con el fin de conseguir un aprendizaje más significativo y duradero. Todas las personas cumplimentaron la prueba previa y posterior. Hubo personas voluntarias para trabajar prácticas en todos los contenidos de la asignatura. El ambiente creado en la clase fue bueno, de colaboración y respeto por quien tenía el turno de intervención. El aprendizaje fue en su mayoría significativo. En intervenciones futuras, se espera mejorar la calidad y carácter novedoso de las propuestas planteadas por el alumnado.

Palabras Clave: Ciclo de Mejora en el Aula, Psicometría, práctica, aprendizaje significativo, participación activa.

\section{Abstract}

Usually, the students of the subject Psychometrics (English group) that forms part of the third course of the Degree in Psychology, dedicated practical classes to solve exercises proposed by teachers and participate in the correction of these, in a situation where it was the teacher who led the situation. The objective of this Cycle of Improvement in the Classroom was to encourage student participation, both in the preparation of exercises and in the resolution of these, in order to achieve a more meaningful and lasting learning. All people completed the pre and post test. There were volunteers to work practices in all the contents of the subject. The atmosphere created in the class was good, of collaboration and respect for who had the turn of intervention. The learning was mostly significant. In future interventions, it is expected to improve the quality and novel nature of the proposals made by students.

Keywords: Cycle of Improvement in the Classroom, Psychometrics, practice, meaningful learning, active participation.

Jornadas de Formación e Innovación Docente del Profesorado | № 2 (2019)

Esta obra se distribuye con la licencia Creative Commons

Reconocimiento-NoComercial-SinObraDerivada $\quad 4.0$ Internacional (CC BY-NC-ND 4.0.) 


\section{Contexto docente}

Psicometría es una asignatura obligatoria de 30 del Grado en Psicología. En pocas palabras, se podría decir que el objetivo de dicha asignatura es aprender a construir instrumentos que midan aspectos psicológicos de las personas, como la autoestima o las habilidades sociales, por ejemplo. A dichos instrumentos se les denomina genéricamente "tests psicológicos". El Ciclo de Mejora en el Aula (CIMA) que a continuación se presenta se realizó en el grupo 1, impartido en inglés. Una de las principales características que diferencia a este grupo del resto es que formalmente no puede tener más de 40 matriculados mientras que, en los otros grupos, puede haber el doble. Otra característica es que suele haber alumnado que viene de universidades de otros países para cursar un cuatrimestre o el curso completo. Los materiales escritos están en inglés, al igual que los exámenes. La docencia también se imparte en este idioma.

En las clases impartidas habitualmente, el alumnado se encontraba en la página web de la asignatura los materiales tanto para las clases teóricas como para las prácticas. En clase se utilizaban como base de las explicaciones teóricas unas diapositivas, mientras que en prácticas el alumnado realizaba los ejercicios propuestos y, pasados unos minutos, se ponían en común para resolver las posibles dudas que pudieran surgir.

Al ser de $3^{\text {er }}$ curso, el alumnado suele estar bien orientado en cuanto a las características de la Facultad y la metodología utilizada. A veces el alumnado que viene de otras universidades es el que precisa más atención por las diferencias en el idioma, porque a veces no comprenden cómo funciona el horario de clase y no sabe a qué grupo asistir, etc. Todos suelen ser cordiales y atienden en clase, aunque a veces hay que pedir algo de silencio porque ya no se oye a quien está exponiendo (especialmente a última hora).

Jornadas de Formación e Innovación Docente del Profesorado | № 2 (2019) 
Las aulas de las clases teóricas tenían sillas de pala, lo que permitía la movilidad del alumnado para las diferentes situaciones que se daban: trabajos en grupo, atender a alguien que expone o realización de exámenes, por ejemplo. Las clases prácticas se realizaron en aulas informatizadas. Por el número de matriculados, cada persona podía utilizar un ordenador (no tenían que compartir).

En el CIMA anterior, se potenció la implicación del alumnado en base a la autocorrección de sus ejercicios (Sanduvete, 2018). Continuando con el mismo principio didáctico (García-Pérez \& Porlán, 2017), en este CIMA se propuso por parte del alumnado la realización de los enunciados de ejercicios y casos prácticos para que el resto del alumnado los resolviera, y su posterior corrección. Además, de este modo se trató también de que los ejercicios fueran más significativos y motivadores para el alumnado y la adquisición de un rol más activo por parte del alumnado. En las autocorrecciones del CIMA anterior, a veces se daban por erróneos procedimientos alternativos correctos. Para evitar que esto pudiera volver a ocurrir, la persona que preparaba el ejercicio pensaba todas las posibles alternativas de solución. El grupo de clase complementaría aportando otras posibles alternativas que no se hubieran tenido en cuenta inicialmente.

Si bien es cierto que el CIMA anterior se realizó en una asignatura diferente, no se previeron problemas para la extrapolación de lo aprendido entonces a la nueva situación, dado que existían muchas similitudes, como por ejemplo: (a) en ambos casos, el alumnado era del grupo de inglés, con sus características particulares previamente mencionadas (similar número de matriculados, por ejemplo); (b) ambas asignaturas son del Área de Metodología y tienen un fuerte componente estadístico; y (c) las aulas de entonces tenían las mismas características en ambos casos.

Jornadas de Formación e Innovación Docente del Profesorado | № 2 (2019)

Esta obra se distribuye con la licencia Creative Commons 


\section{Mapa de contenidos y secuencia de actividades}

Siguiendo las recomendaciones de García-Díaz, Porlán y Navarro (2017) para su elaboración, el mapa de contenidos que se pretende que los estudiantes aprendan y las preguntas-clave asociadas se presentan en la Figura 1.

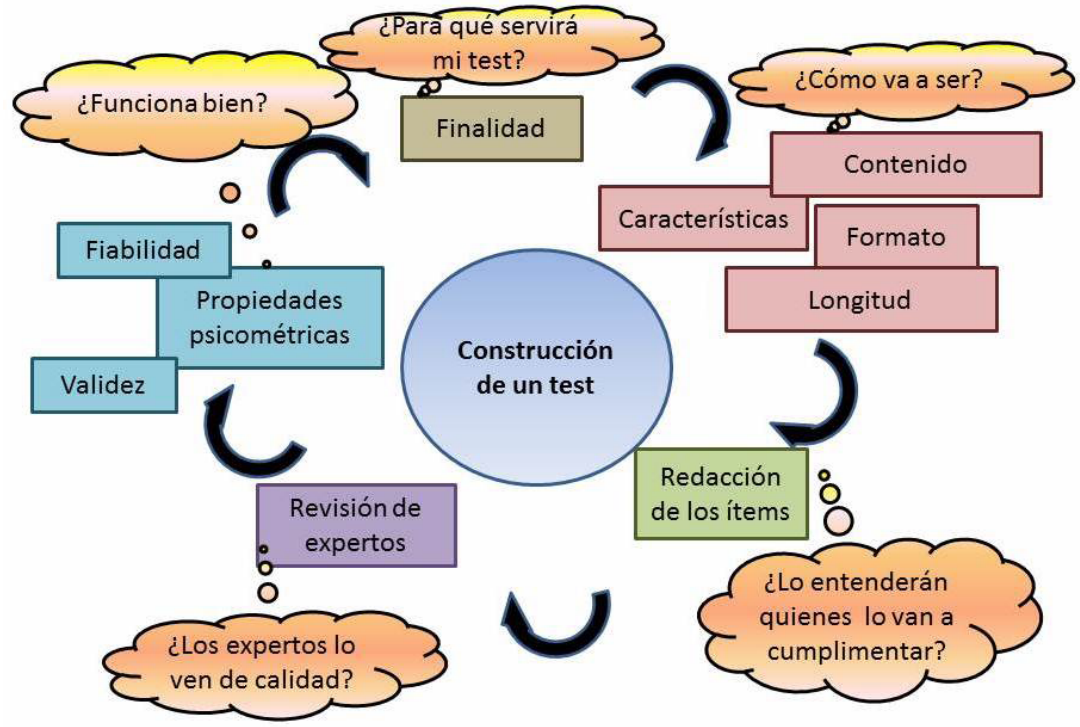

Figura 1. Mapa de contenidos y preguntas-clave.

Uno de los aspectos más relevantes y posiblemente más dificiles de comprender en psicometría es que las fases de construcción de un test no son lineales. El test se va elaborando pero, a cada paso que se da, se valora si el procedimiento de construcción va bien y, en caso de que se detecten defectos, se vuelve a la fase anterior para su mejora (antes de acabar del todo para darse cuenta de que el test no mide correctamente lo que pretendía medir). Esta retroalimentación se representa en la Figura 2 a través de las flechas y con el formato circular de estructuración de los contenidos.

No se prescindió de ninguno de los contenidos, puesto que todos son necesarios para la elaboración de un test; sin embargo, en función de que se fue avanzando en el

Jornadas de Formación e Innovación Docente del Profesorado | № 2 (2019)

Esta obra se distribuye con la licencia Creative Commons 
cuatrimestre, se profundizó más en aquellos aspectos que resultaron dificiles para el alumnado, puesto que los más sencillos pudieron interiorizarlos con el trabajo continuado personal, sin necesidad de tanta ayuda externa.

A continuación, se presenta la actividad que realizó el alumnado:

Os animamos a que elaboréis ejercicios y/o casos prácticos para la asignatura, con la finalidad de motivar a vuestra/os compañeras/os de clase a practicar los contenidos. Se trata de un trabajo voluntario que recibirá recompensa en la calificación final de la asignatura. Para participar, sigue los siguientes pasos:

1. Envía un correo electrónico a sussancha@us.es, indicando qué temática escoges para elaborar tu trabajo, de entre las siguientes:

a) Reconocimiento de los usos posibles que tiene un test.

b) Reconocimiento de los distintos contenidos que puede tener un test.

c) Reconocimiento de los distintos tipos de ítems.

d) Cómo calcular la longitud ideal de un test.

e) Determinar si los ítems están bien o mal redactados.

f) Validez de contenido (Osterlind).

g) Fiabilidad: Cronbach

h) Fiabilidad: KR20

i) Fiabilidad KR21

j) Validez de constructo.

2. Elabora un ejercicio llamativo, que pueda interesar a tus compañeros/as.

3. Resuelve tu propio ejercicio. Revisa que lo hiciste bien.

4. Envialo a sussancha@us.es. Espera confirmación.si no hay respuesta en dos dias, por favor, insiste (por si el correo electrónico ha fallado).

Jornadas de Formación e Innovación Docente del Profesorado | № 2 (2019)

Esta obra se distribuye con la licencia Creative Commons 
5. Este paso es optativo, para las/os más valientes. Una semana después de recibir feedback de la profesora, propondrás la realización de tu ejercicio a la clase y, pasados unos minutos, lo expondrás y resolverás las posibles dudas que puedan surgir.

\section{Modelo metodológico seguido}

En el aspecto en que me centré, el modelo metodológico habitual consistía en que el alumnado se encontraba elaborados los materiales de prácticas, resolvía los ejercicios y casos prácticos elaborados por el profesorado, y luego se corregían con una puesta en común en clase.

Mi modelo metodológico ideal implicaba que el alumnado realizara todos los ejercicios, de tal manera que éstos estuvieran centrados en temas en los que dicho alumnado estuviera interesado, por lo que sería un material significativo para los participantes. Suponiendo esto, la motivación del alumnado por trabajarlos habría de ser mayor (Polanco-Hernández, 2005). Además, tener que inventar casos prácticos les obligaría a tener que entender en profundidad el contenido teórico, o no sabrían plantear un ejercicio y resolverlo.

El modelo metodológico que vi como posible fue un híbrido entre los dos anteriores. Se utilizarían ejercicios previos ya elaborados, principalmente por el escaso tiempo del que se disponía para trabajar todo el temario de la asignatura, que es realmente amplio. Sin embargo, se promovería que cada matriculado propusiera un ejercicio de elaboración propia a resolver por los demás.

La Figura 2 muestra gráficamente en qué consistió el modelo metodológico seguido.

Jornadas de Formación e Innovación Docente del Profesorado | № 2 (2019)

Esta obra se distribuye con la licencia Creative Commons 


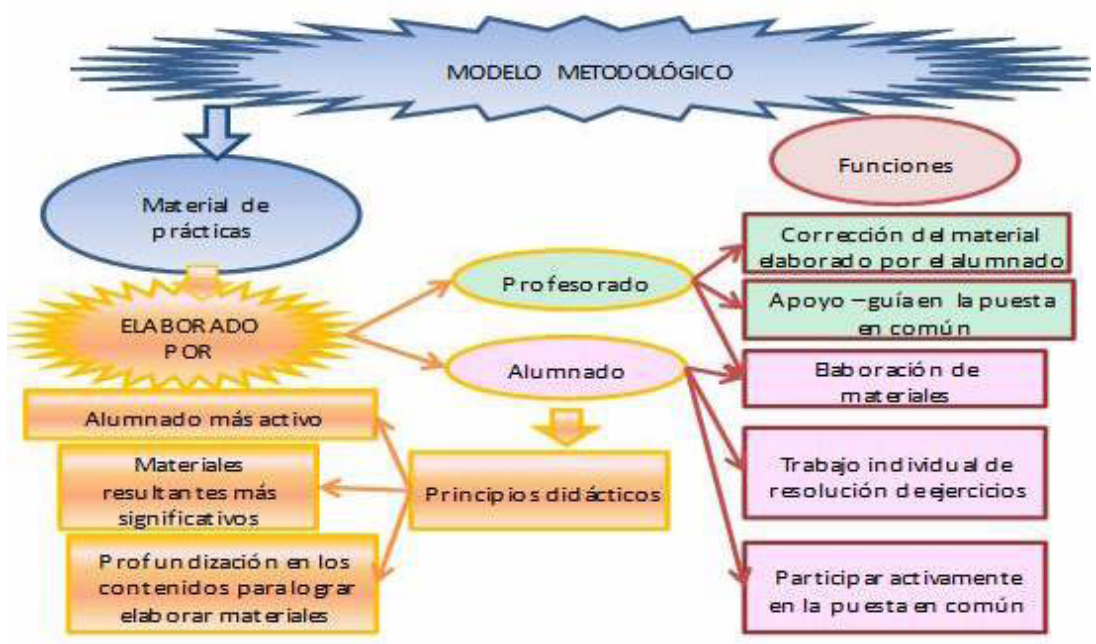

Figura 2. Modelo metodológico seguido.

\section{Relato resumido de las sesiones}

El alumnado se mostró voluntario para cubrir contenidos específicos vía correo electrónico (la mayoría) o personalmente en clase o en horario de tutorías.

En todas las sesiones, los pasos a seguir fueron los siguientes: (a) el alumnado propuso un ejercicio; (b) se dejó unos 10 minutos para que todos en clase lo resolvieran; (c) quien propuso el ejercicio expuso su solución, tratando de que el resto de alumnado participara con una actitud activa; (d) quien propuso el ejercicio resolvió las dudas que fueron surgiendo; y (e) el profesorado intervino (si fue necesario) para aclarar cuestiones que pudieron quedar dudosas y / o para incidir en algún aspecto relevante que pudo ser pasado por alto.

La Tabla 1 presenta, de manera anónima, al alumnado que participó en cada contenido y los comentarios abiertos que se recogieron, en su caso, más alguna otra información relevante para el seguimiento (fecha y grupo).

Jornadas de Formación e Innovación Docente del Profesorado | № 2 (2019)

Esta obra se distribuye con la licencia Creative Commons 
Tabla 1. Seguimiento hecho del alumnado participante.

\begin{tabular}{|c|c|c|c|c|}
\hline Participante & Contenidos tratados & Fecha & Grupo & Observaciones \\
\hline A & Finalidad del test & $01 / 10$ & Martes & Intervención sobresaliente \\
\hline B & Características del test & $01 / 10$ & Martes & Intervención adecuada \\
\hline C & Contenido del test & $01 / 10$ & Martes & No explicó en voz alta \\
\hline $\mathrm{D}$ & $\begin{array}{c}\text { Finalidad y características } \\
\text { del test }\end{array}$ & $03 / 10$ & Jueves & $\begin{array}{c}\text { Intervención sobresaliente. } \\
\text { No lleva notas en papel }\end{array}$ \\
\hline$E$ & Contenido del test & $03 / 10$ & Jueves & Intervención sobresaliente \\
\hline $\mathrm{F}$ & Revisión de expertos & $08 / 10$ & Martes & Intervención sobresaliente \\
\hline $\mathrm{G}$ & Redacción de los ítems & $08 / 10$ & Martes & $\begin{array}{c}\text { No supo responder a todas } \\
\text { las preguntas }\end{array}$ \\
\hline $\mathrm{H}$ & Validez & $15 / 10$ & Martes & Intervención adecuada \\
\hline I & Revisión de expertos & $10 / 10$ & Jueves & Intervención sobresaliente \\
\hline J & $\begin{array}{c}\text { Análisis métrico de los } \\
\text { ítems }\end{array}$ & $22 / 10$ & Martes & Intervención adecuada \\
\hline $\mathrm{K}$ & Redacción de los ítems & $17 / 10$ & Jueves & Intervención adecuada \\
\hline $\mathrm{L}$ & Validez & $24 / 10$ & Jueves & Intervención adecuada \\
\hline M & $\begin{array}{c}\text { Análisis métrico de los } \\
\text { ítems }\end{array}$ & $05 / 11$ & Martes & Intervención adecuada \\
\hline$N$ & $\begin{array}{c}\text { Análisis métrico de los } \\
\text { ítems }\end{array}$ & $07 / 11$ & Jueves & Intervención adecuada \\
\hline 0 & $\begin{array}{c}\text { Análisis métrico de los } \\
\text { ítems }\end{array}$ & $14 / 11$ & Jueves & $\begin{array}{c}\text { Intervención altamente } \\
\text { notable }\end{array}$ \\
\hline$P$ & Fiabilidad & $14 / 11$ & Jueves & $\begin{array}{c}\text { Intervención sobresaliente. } \\
\text { Diferentes alternativas } \\
\text { de solución para un solo } \\
\text { ejercicio }\end{array}$ \\
\hline Q & Fiabilidad & $19 / 11$ & Martes & Intervención sobresaliente \\
\hline
\end{tabular}

\section{Evaluación del aprendizaje del alumnado a lo largo del CIMA}

El martes 23 de septiembre, día de la presentación de la asignatura, y el martes 12 de noviembre, tres días antes de la finalización del CIMA, se aplicó el siguiente cuestionario, como pre-test y post-test respectivamente, que tocaba todos los contenidos a tratar y se basaba en las preguntas-clave propuestas:

Jornadas de Formación e Innovación Docente del Profesorado | № 2 (2019)

Esta obra se distribuye con la licencia Creative Commons Reconocimiento-NoComercial-SinObraDerivada $\quad 4.0$ Internacional (CC BY-NC-ND 4.0.) 
Por favor, responde a las siguientes preguntas. Tu participación activa redundará positivamente en la calificación final de la asignatura.

1. ¿Qué miden los tests psicológicos?

2. Enumera los tipos de ítems que hay.

3. ¿Hay un número ideal de ítems para un test? ¿Por qué?

4. "No creo que nada cambie". ¿Mejorarías este ítem? ¿Cómo?

5. ¿Para qué usarías el índice de Osterlind?

6. Define fiabilidad. ¿Por qué es importante?

7. Define validez. ¿Por qué es importante?

Se propusieron preguntas abiertas porque se considera que, con este formato, se podría profundizar en mayor medida en el tipo de respuesta que daba el alumnado y en su grado de conocimiento (Rivero y Porlán, 2017).

Se pidió al alumnado que escribiera su nombre en el cuestionario porque se valoraría positivamente para la calificación final de la asignatura: (a) la mera participación en los cuestionarios y los ejercicios; (b) la calidad de las respuestas en el cuestionario final, en los ejercicios y en la exposición en clase.

Estas puntuaciones se sumaron a las del examen final para obtener la calificación global. Con esto, se persiguieron varios fines: (a) animar a la participación en la actividad propuesta y su evaluación; (b) favorecer que el alumnado fuera estudiando poco a poco, en lugar de dejarlo todo para el momento del examen final; (c) dar con las calificaciones continuas la posibilidad de no jugárselo todo en el examen final.

La información recogida antes del CIMA se utilizó para la elaboración de la escalera inicial de aprendizaje, que permitió clasificar qué tipo de respuesta dio el alumnado, además de detectar en qué aspectos se hacía más

Jornadas de Formación e Innovación Docente del Profesorado | № 2 (2019)

Esta obra se distribuye con la licencia Creative Commons 
necesario incidir en función de sus conocimientos previos. La escalera de aprendizaje final se comparó con la inicial en base al porcentaje de tipo de respuesta dada. Se esperaba que un porcentaje más alto de participantes diera respuestas de mayor calidad al final del CIMA en comparación con el principio. Efectivamente, esto fue lo que ocurrió. La Tabla 2 muestra los resultados obtenidos: los porcentajes de participantes que responden a cada respuesta en cada pregunta antes y después del CIMA y, en la última columna, el valor del estadístico $X^{2}$ de McNemar junto con sus grados de libertad y la significación obtenida.

Tabla 2. Resultados obtenidos en el cuestionario (medida antes y después del CIMA)

\begin{tabular}{|c|c|c|c|c|c|}
\hline № & Ítem & Respuestas $(N=25)$ & $\%$ pre & $\%$ post & $\mathrm{X}^{2} / g l / p$ \\
\hline 1 & $\begin{array}{l}\text { Qué miden los } \\
\text { tests }\end{array}$ & $\begin{array}{l}\text { No contesta } \\
\text { Responde con ejemplo } \\
\text { (inteligencia) } \\
\text { Responde parcialmente } \\
\text { Responde completamente }\end{array}$ & $\begin{array}{c}4 \\
28 \\
52 \\
16\end{array}$ & $\begin{array}{l}--- \\
8 \\
24 \\
68\end{array}$ & $\begin{array}{c}15 \\
3 \\
.002\end{array}$ \\
\hline 2 & Tipos de ítems & $\begin{array}{l}\text { Enumera } 0-1 \text { tipos } \\
\text { Enumera 2-4 tipos } \\
\text { Enumera 5-6 tipos } \\
\text { Enumera } 7-8 \text { tipos }\end{array}$ & $\begin{array}{l}88 \\
12 \\
---\end{array}$ & $\begin{array}{l}32 \\
32 \\
12 \\
24\end{array}$ & $\begin{array}{c}14 \\
5 \\
.016\end{array}$ \\
\hline 3 & $\begin{array}{l}\text { Número de } \\
\text { ítems }\end{array}$ & $\begin{array}{l}\text { No responde } \\
\text { Respuesta errónea } \\
\text { Correcto sin razonar }\end{array}$ & $\begin{array}{l}16 \\
11 \\
10 \\
--\end{array}$ & $\begin{array}{l}12 \\
64 \\
--- \\
24\end{array}$ & $\begin{array}{c}14.33 \\
3 \\
.002\end{array}$ \\
\hline 4 & $\begin{array}{l}\text { Mejora ítem } \\
\text { con doble } \\
\text { negación }\end{array}$ & $\begin{array}{l}\text { No responde } \\
\text { Respuesta errónea } \\
\text { Respuesta correcta incompleta } \\
\text { Respuesta correcta bien } \\
\text { razonada }\end{array}$ & $\begin{array}{c}4 \\
40 \\
48 \\
8\end{array}$ & $\begin{array}{l}--- \\
36 \\
64\end{array}$ & $\begin{array}{c}15.4 \\
3 \\
.002\end{array}$ \\
\hline 5 & $\begin{array}{l}\text { Uso de } \\
\text { Osterlind }\end{array}$ & $\begin{array}{l}\text { No contesta } \\
\text { Describe indice parcialmente } \\
\text { Explica uso parcialmente } \\
\text { Explica uso correctamente }\end{array}$ & $\begin{array}{c}84 \\
8 \\
8 \\
---\end{array}$ & $\begin{array}{c}12 \\
8 \\
24 \\
56\end{array}$ & $\begin{array}{c}20 \\
5 \\
.001\end{array}$ \\
\hline 6 & $\begin{array}{l}\text { Definición } \\
\text { importancia } \\
\text { fiabilidad }\end{array}$ & $\begin{array}{l}\text { No responde } \\
\text { Descripción pobre } \\
\text { Definición parcial } \\
\text { Definición de componentes }\end{array}$ & $\begin{array}{l}36 \\
28 \\
24 \\
12\end{array}$ & $\begin{array}{c}4 \\
8 \\
28 \\
60\end{array}$ & $\begin{array}{c}18 \\
6 \\
.006\end{array}$ \\
\hline
\end{tabular}

Jornadas de Formación e Innovación Docente del Profesorado | № 2 (2019)

Esta obra se distribuye con la licencia Creative Commons 


\begin{tabular}{|c|l|l|c|c|c|}
\hline \multirow{7}{*}{7} & Definición & No responde & 12 & --- & 19 \\
& importancia & Descripción pobre & 40 & 4 & 5 \\
& validez & Definición parcial & 36 & 36 & .002 \\
\hline
\end{tabular}

En todas las ocasiones se obtuvo una mejora significativa $(p<.05)$ al comparar el conocimiento antes del CIMA y después. Las respuestas fueron tras el CIMA más correctas, más precisas y completas.

\section{Evaluación del CIMA puesto en práctica}

La participación en la cumplimentación de las pruebas previa y posterior al CIMA fue total (todas las personas matriculadas participaron en ambos momentos).

En la actividad (exposición de ejercicios), participó un total de 17 estudiantes, lo que supuso el 68\% del total de personas matriculadas en el grupo. Las clases prácticas eran semanales. Cada semana, entre dos y cinco participantes diferentes expusieron ejercicios. Hubo un muy buen ajuste entre el contenido, la calendarización y la participación, puesto que apenas hubo contenido que no expusiera algún estudiante, y no hubo ningún estudiante que, teniendo interés de participar, no pudiera hacerlo por falta de tiempo o de contenido.

A lo largo del proceso, no hubo incidencias importantes a comentar. Como aspectos positivos, destacaría: (a) el alto grado de participación en los cuestionarios y las actividades; (b) la actitud colaborativa de los compañeros cuando alguien exponía, guardando silencio cuando la situación lo requería y participando con sus respuestas y comentarios cuando así se les solicitaba; (c) todos los contenidos de la asignatura, ya fueran fáciles o dificiles, tuvieron personas voluntarias para su exposición; y (d) la asistencia a clase fue muy elevada. Como cuestiones negativas, destacaría: (a) un caso aislado donde una alumna,

Jornadas de Formación e Innovación Docente del Profesorado | № 2 (2019)

Esta obra se distribuye con la licencia Creative Commons 
que se propuso para resolver determinado ejercicio, en el momento aún no lo tenía preparado, y la exposición no fue muy acertada (cometió errores que hubieron de corregirse); y (b) los ejercicios propuestos no fueron novedosos; fueron prácticamente iguales que los ya propuestos en la web por el profesorado.

Adicionalmente, se recogió información acerca de la opinión del alumnado sobre el CIMA con las siguientes preguntas:

1. Valora de 1 (totalmente inútil) a 5 (totalmente útil) la actividad realizada.

2. ¿Se podría mejorar para cursos venideros? ¿Cómo?

Se obtuvo una media de 3.9 que, sobre 5 , se puede interpretar como que el alumnado consideró bastante útil el CIMA. Nadie propuso mejoras para intervenciones futuras.

En base a toda la información recogida, las propuestas de mejora para próximas intervenciones son: (a) forzar al menos una consulta previa a la exposición, ya sea por correo electrónico o en persona, para evitar que se cometan errores importantes en la exposición en clase; y (b) premiar la imaginación y el carácter novedoso en los ejercicios elaborados.

Jornadas de Formación e Innovación Docente del Profesorado | № 2 (2019) 


\section{Referencias bibliográficas}

García-Díaz, E., Porlán, R., \& Navarro, E. (2017). Los fines y los contenidos de enseñanza. En R. Porlán (Coord.), Enseñanza Universitaria. Cómo mejorarla (pp. 93-104). Madrid, España: Morata.

García-Pérez, F. y Porlán, R. (2017). Los principios didácticos y el modelo didáctico personal. En R. Porlán (Coord.), Enseñanza Universitaria. Cómo mejorarla (pp. 93-104). Madrid, España: Morata.

Polanco-Hernández, A. (2005). La motivación en los estudiantes universitarios. Actualidades Investigativas en Educación, 5(2), 1-13.

Rivero, A, y Porlán, R. (2017). La evaluación en la enseñanza universitaria. En R. Porlán (Coord.), Enseñanza Universitaria. Cómo mejorarla (pp. 73-91). Madrid, España: Morata.

Sanduvete, S. (2018). Autocorrección para el aprendizaje y el fomento de la autonomía. En V Jornadas de Docencia Universitaria (pp. 816-831). Sevilla: Instituto de Ciencias de la Educacion de la Universidad de Sevilla. Disponible en http://dx.doi.org/10.12795/JDU.2018.i01.46

Jornadas de Formación e Innovación Docente del Profesorado | № 2 (2019)

Esta obra se distribuye con la licencia Creative Commons 